\title{
Entwicklung und Anwendung einer elektronischen Zunge zum Nachweis organischer Säuren in Biogasgülle
}

\author{
Thomas Nacke ${ }^{1}$, Andreas Barthel', Daniel Martin ${ }^{1}$, Olaf Schröder ${ }^{2}$, Yahor Zaikou ${ }^{1}$ \\ ${ }^{1}$ iba e. V. Heilbad Heiligenstadt, Deutschland \\ ${ }^{2}$ Pattern Expert. Leipzig, Deutschland \\ Kontakt: thomas.nacke@iba-heiligenstadt.de
}

\begin{abstract}
Zusammenfassung:
Um Prozessstörungen in Biogasanlagen (BGA) und Fermentationsverläufen schneller zu erkennen, werden online und feldtaugliche Analysenmethoden benötigt. Durch die Anwendung einer elektronischen Zunge können online die Anteile flüchtiger organischer Säuren (FOS) mittels impedanzspektroskopischer Messmethoden in Biogasgüllen erfasst werden. Diese elektrochemische Messmethode basiert auf einem Multielektrodenarray mit unterschiedlichen Elektrodenmaterialien, einem impedanzspektroskopischen Messsystem und der Anwendung von multivariaten statistischen Datenauswertungen (MSD) zur Kalibration und quantitativen Ausgabe der Messergebnisse.
\end{abstract}

Schlagwörter: Impedanzspektroskopie, elektronische Zunge, organische Säuren, Biogasgülle

\section{Motivation und Einleitung}

Durch die Co-Vergärung von verschiedenen nachwachsenden Rohstoffen (NaWaRo) mit unterschiedlichen Stoffeigenschaften (Anteile von Wasser, Trockensubstanz (TS), organische TS (oTS), Fetten, Proteinen, Kohlenhydraten u.a.) kommt es im Fermenter zu räumlichen und zeitlichen Heterogenitäten, welche die Prozessstabilität und damit die Wirtschaftlichkeit der BGA beeinflussen.

Tabelle 1: Anteile von FOS in Biogasanlagen [1]

\begin{tabular}{|l|c|}
\hline \multicolumn{1}{|c|}{ Säure, Art } & Anteil in g/L \\
\hline Acetate, Essigsäure & $0,5-15,6$ \\
\hline Propionate, Propionsäure & $0,2-5,9$ \\
\hline Iso-butyrate & $0,0009-2,8$ \\
\hline Butyrate, Buttersäure & $0,2-5,7$ \\
\hline Iso-valerate & $0,0009-2,7$ \\
\hline Valerate, Valeriansäure & $0,0009-2,8$ \\
\hline n-hexanoate Hexansäure & $0,0005-1,3$ \\
\hline Gesamtanteil von FOS & ca. 5,4 \\
\hline
\end{tabular}

Eine grundlegende Voraussetzung zur Realisierung einer Prozessoptimierung besteht in der Entwicklung von robusten und marktgerechten Online-Prozessmesssystemen, mit welchen in Echtzeit der Prozessverlauf einer anaeroben Biogasfermentation erfasst werden kann. Aktuell werden Messwerte von Stoffeigenschaften an BGA meist nur punktuell nach einer laboranalytischen Untersuchung erfasst. Ein optimierter Biogasprozess hinsichtlich einer bedarfsgerechten und stabilen Gasproduktion kann mit Offline-Laboranalysatoren nicht erreicht werden. Die Bestimmung von FOS mit Methoden der lonenchromatographie und Hochleistungsflüssigkeitschromatographie (HPLC: High Performance Liquid Chromatography) im Laborbetrieb entspricht dem allgemeinen Standard der Technik[1]. Neue Messtechnologien zum Prozessmonitoring von BGA müssen noch für die erfolgreiche Anwendung auf dem Biogassektor getestet und angepasst werden [2].

Durch die Entwicklung und Anwendung einer elektronischen Zunge, engl. "Electronic Tongue" $(E T)$, zur Online-Bestimmung von FOS direkt in Biogasgüllen soll ein Beitrag zur Überwindung dieser Defizite geleistet werden. Ein allgemeiner Messablauf für ET zeigt die Abbildung 1.

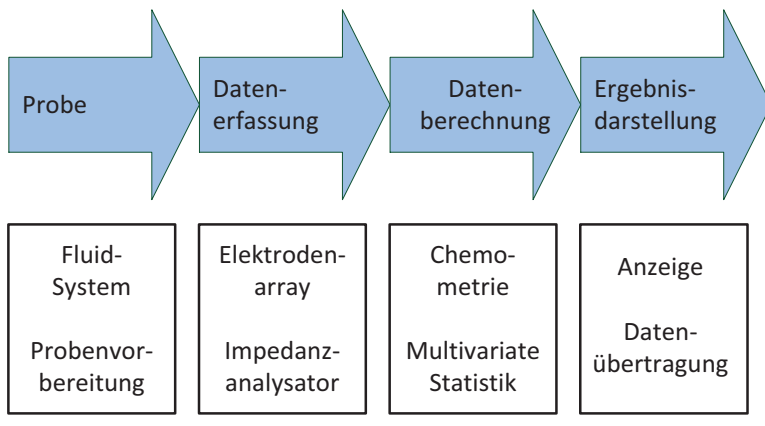

Abbildung 1: Charakteristische Messanordnung einer ET mit impedanzspektroskopischer Messtechnik

In diesem Beitrag werden die bisherigen Ergebnisse eines noch bis Ende 2016 laufenden Verbundprojektes vorgestellt.

Bisherige Erkenntnisse auf dem Gebiet der ET sollen für die Entwicklung einer feldtauglichen Messtechnik weiterentwickelt und zur Anwendung gebracht werden $[3,4,5]$. 


\section{Grundprinzip elektronischer Zungen}

Seit Beginn der 90er Jahre wird versucht, die beeindruckenden Geschmacksempfindlichkeiten der tierischen und menschlichen Zunge und Nase mit elektronischen Sensoren nachzubilden. Dabei kommen bisher die verschiedensten elektrochemischen Messmethoden, wie Voltametrie, Amperometrie und Impedanzspektroskopie zur Anwendung. Hauptanwendungsgebiete sind Untersuchungen zur Herkunft, zu Inhaltsstoffen, Verunreinigungen und der Qualität in der Lebensmitteltechnik, in der Wasser- und Trinkwasseranalyse und in der Medizintechnik, z.B. zur Detektion von Krankheitserregern [3]. Trotz des erhöhten technischen Aufwandes findet die elektrische Impedanzspektroskopie (EIS) für Anwendungen der ET verstärktes Interesse. Die Vorteile bestehen in der Anwendung kleinerer Anregungsspannungen, mit weniger Einfluss auf die Elektrodeneigenschaften und deren Standzeiten. Weiterhin in der Erfassung der frequenzabhängigen komplexen konduktiven und dielektrischen Eigenschaften in Abhängigkeit von der Wechselwirkung zwischen den Elektroden und den zu untersuchenden Elektrolyten [6].

Viele Elektroden (Elektrodenarray) aus unterschiedlichen Metallen mit und ohne Beschichtungen bilden dabei die künstlichen „Geschmacksknospen".

Wird eine Metallelektrode in eine Flüssigkeit (Elektrolyt) getaucht, so besteht bei den Metallionen der Elektroden das Bestreben, in Lösung zu gehen. Die Ionen des Elektrolyten versuchen wiederum, mit den Metallelektroden an der Oberfläche eine Verbindung einzugehen [7]. Dies führt an der Grenzfläche zwischen Elektrode und Elektrolyt zum Aufbau einer Ladung in Form einer Doppelschicht (Abbildung 2).

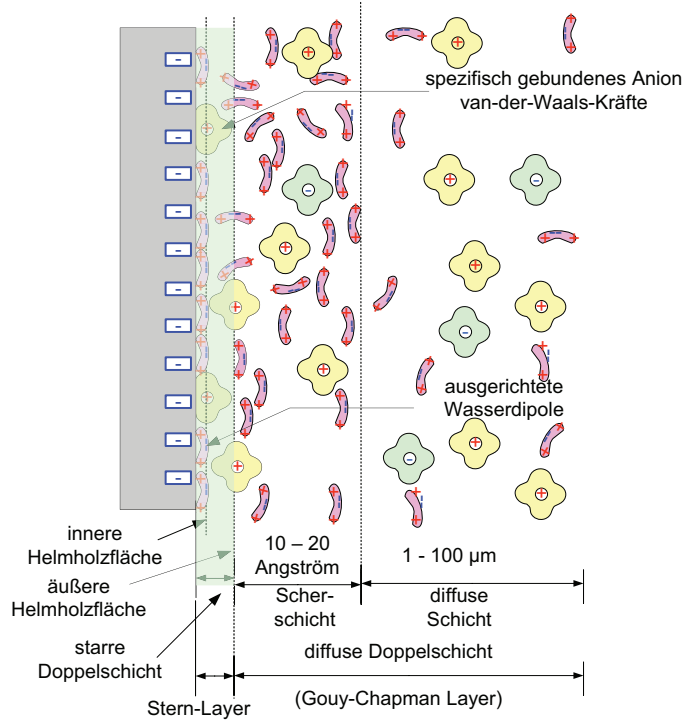

Abbildung 2: Modell einer elektrochemischen Doppelschicht
Durch die oberflächenspezifische Ausprägung einer elektrochemischen Doppelschicht an den Elektrodenoberflächen, in Abhängigkeit von den in den Flüssigkeiten enthaltenen lonen (Anionen und Kationen), kann mit den oben genannten elektrochemischen Methoden ein von dem Elektrodenmaterial abhängiges Messsignal generiert werden.

Die EIS ist eine etablierte Messmethode, um die frequenzabhängige Ausbildung der Doppelschicht messtechnisch zu beschreiben. Hierzu wird an das Elektrodensystem im Frequenzbereich von einigen $\mathrm{mHz}$ bis ca. $100 \mathrm{kHz}$ eine Generatorspannung von $10 \mathrm{mV}$ bis $100 \mathrm{mV}$ angelegt. Durch eine phasengenaue Messung der Strom- und Spannungswerte an den Elektroden kann die frequenzabhängige komplexe Impedanz gemessen werden.

Die Abbildung 3 zeigt das Impedanzspektrum einer Ir-Elektrode in Abhängigkeit von der Konzentration einer Propionsäure in einem Phosphatpuffer.

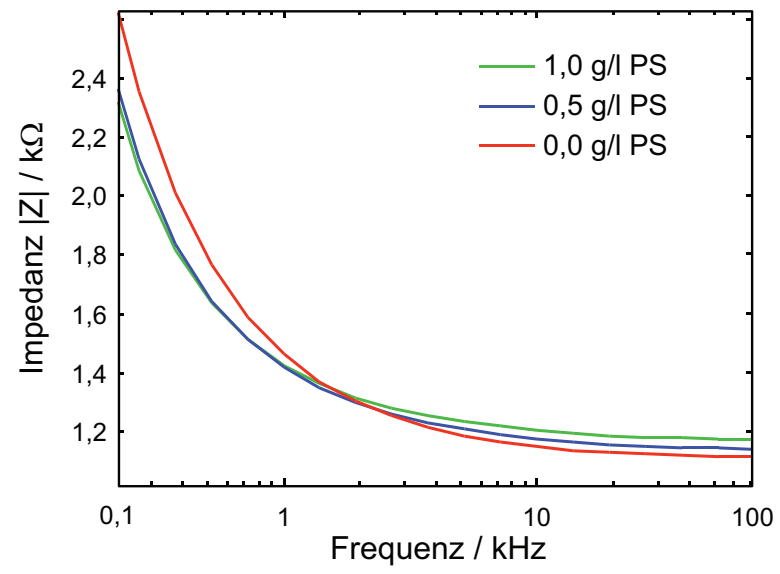

Abbildung 3: Typisches Impedanzspektrum, Iridiumelektrode in einem Elektrolyten aus Phosphatpuffer und Propionsäure

Zur Interpretation der Spektren bezüglich der Oberflächenreaktionen an den Elektroden lassen sich Ersatzschaltbilder erstellen. Dabei wird häufig ein Ersatzschaltbild nach Randles eingesetzt. Es besteht aus einem Serienwiderstand $\mathrm{R}_{\mathrm{s}}$ (stellt die Leitfähigkeit des Elektrolyten zwischen den Elektroden dar) und einer Parallelschaltung einer Kapazität $\mathrm{C}_{\mathrm{dl}}$ (Doppelschichtkapazität) sowie einem aktiven Ladungstransferwiderstand in Reihe mit einem spezifischen Diffusionselement W (Warburgimpedanz). Die Doppelschichtkapazität wird oft durch das komplexere konstante Phasen Element (CPE: Constant Phase Element) ersetzt $[8,9]$.

In Abhängigkeit von der lonenkonzentration können im niederen Frequenzbereich bis ca. $10 \mathrm{kHz}$ die Eigenschaften der elektrischen Doppelschicht abgebildet und mit einer geeigneten Ersatzschaltung simuliert werden. 
$\mathrm{Ab}$ einem Frequenzbereich von größer $10 \mathrm{kHz}$ werden die Bulkeigenschaften erfasst und können vereinfacht mit einer parallelen Ersatzschaltung, bestehend aus einem Widerstand und einem Kondensator, simuliert werden. Der Widerstand steht für die Leitfähigkeit und der Kondensator für die Permittivität des Elektrolyten.

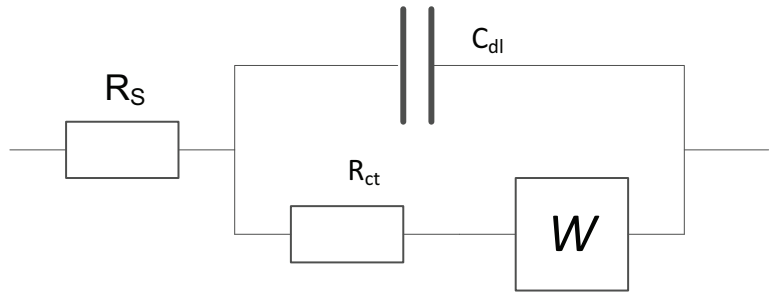

Abbildung 4: Ersatzschaltbild zur vereinfachten Beschreibung der Elektroden- Elektrolytinteraktion.

Somit ist es möglich, mehrere Stoffgruppen gleichzeitig zu analysieren, da zum Beispiel eine reaktive Säure ein anderes Signal als Glukose erzeugt.

\section{Messkonzept}

Durch die komplexe stoffliche Zusammensetzung von realen Biogasgüllen werden bezüglich der Reproduzierbarkeit der Messungen erhöhte Anforderungen an die Probenahme, Referenzmedien sowie an die Kalibration und Datenberechnung auf Basis eines multivariaten Regressionsmodells, gestellt. Die Ansteuerung des gesamten Messplatzes (Abbildung 5) erfolgt auf Grundlage eines am iba in $\mathrm{C}++$ entwickelten Programms.

Für die Datenauswertung mit MSR und Ergebnisdarstellung wurde die Software MATLAB von der MathWorks, Inc. verwendet.

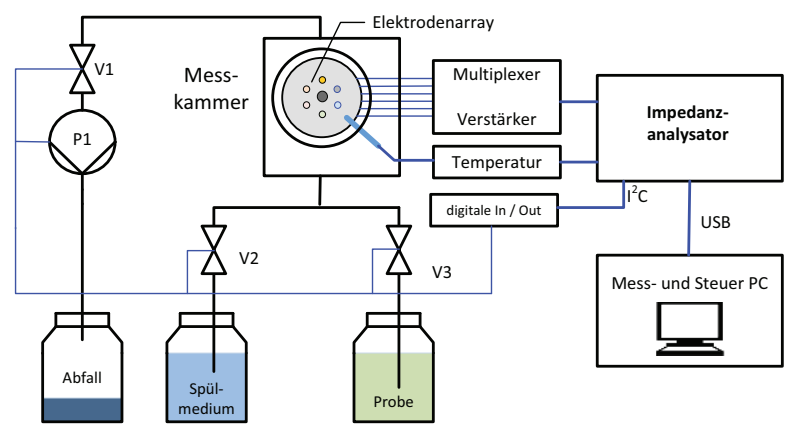

Abbildung 5: Konfiguration des Messablaufs einer ET

\section{Datenerfassung}

Zuerst erfolgte der Aufbau eines universellen elektrochemischen Messplatzes auf Basis eines prozesstauglichen und kostengünstigen Impedanzanalysators (HandyScope HS5 von TiePie) mit einem Multiplexer zur Elektrodenansteuerung als Eigenentwicklung des iba (siehe Abbildung 6).
Der Multiplexer beinhaltet ein, an die frequenzabhängige Widerstandsdynamik (1 $\mathrm{M} \Omega$ bis einigen $\mathrm{k} \Omega$ ) angepasstes Front-End, um den Spannungsabfall und den Stromfluss über die Elektroden mit hoher Auflösung zu messen. Zur Überprüfung und Kalibration der elektronischen Komponenten wird neben den einzelnen Elektroden auch ein Widerstandsnetzwerk angesteuert. Bei Bedarf kann zwischen einer Zwei- oder Drei-Elektrodenkonfiguration umgeschaltet werden. Die Messungen erfolgen in einer auf $20^{\circ} \mathrm{C}$ konstant temperierten Messkammer. Eine Messung wird gestartet, wenn die Probentemperatur $20^{\circ} \mathrm{C}$ mit einer Abweichung von +/$0,05^{\circ} \mathrm{C}$ erreicht hat. Hierzu wird der Temperaturverlauf der Probe mit einem Pt 1000 erfasst. Das Fördervolumen beträgt ca. $15 \mathrm{ml} / \mathrm{min}$ pro Messung. Die Messung erfolgt dann unmittelbar nach Anhalten der Pumpe und Schließen der Ventile im Zu- und Ablauf zur Messkammer.

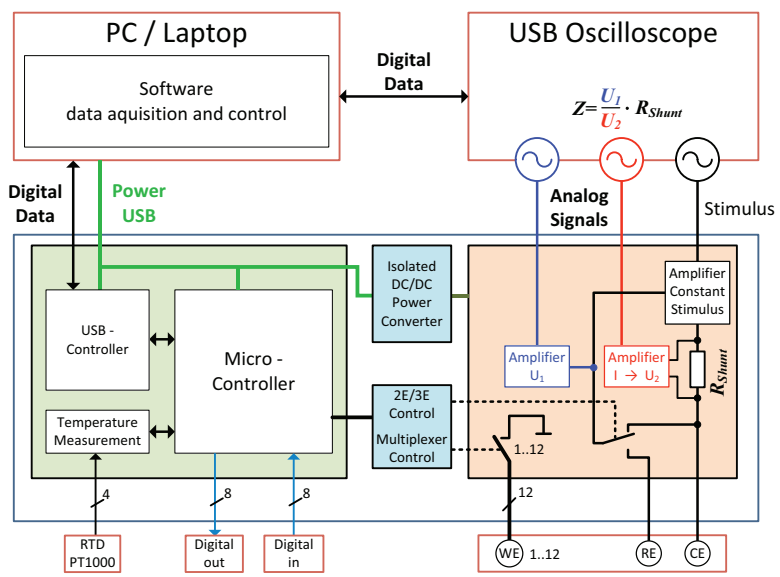

Abbildung 6: Elektrotechnisches Schaltungskonzept mit wahlweiser Nutzung als 2- oder 3-Elektrodensystem, WE- Arbeitselektroden, RE-Referenzelektrode, CEGegenelektrode

In einer kreisförmigen Fluidkammer (Innendurchmesser $22 \mathrm{~mm}$, Volumen ca. $2 \mathrm{ml}$ ) wurden unterschiedliche Metalle (Gold, Iridium, Palladium, Platin, Rhenium, Rhodium) als Punktelektroden (Durchmesser $1 \mathrm{~mm}$ ) ringförmig um eine zentrale Gegenelektrode mit $6 \mathrm{~mm}$ Durchmesser verbaut. Die Elektroden wurden in einer isolierenden Scheibe aus TK-PEEK positioniert und dichtend verklebt. Anschließend wurden zur Erzielung einer guten Standzeit überstehende Metallteile abgeschliffen und mit einem harten Chemiefasertuch und Diamantpolitur die Oberfläche bezüglich einer hohen Planheit und guten Randschärfe bearbeitet.

Erste Messungen erfolgten an Modellmedien. Dabei wurde ein Phosphatpuffer mit den für Biogasmedien typischen flüchtigen Fettsäuren gemischt und impedanzspektroskopisch bei konstanter Temperatur erfasst. 
Aus den Impedanzspektren wurde die frequenzabhängige Kapazität als Parallelschaltung $\left(C_{p}\right)$ eines Widerstandes mit einem Kondensator berechnet (Abbildung 7). Die unterschiedlichen Wechselwirkungen zwischen Elektrodenmaterialien und Elektrolyt sind deutlich an den Spektren zu erkennen. Die Wiederholung der Messung nach ca. $4 \mathrm{~h}$ zeigt eine gute Reproduzierbarkeit. Bezüglich einer langzeitstabilen Messung müssen aber noch geeignete Reinigungsschritte entwickelt und getestet werden.

Als Maß für ein stabiles Messverhalten erfolgt die Bestimmung des Variationskoeffizienten in Prozent von $0,1 \%$ an den Messpunkten des Spektrums zwischen $100 \mathrm{~Hz}-50 \mathrm{kHz}$.

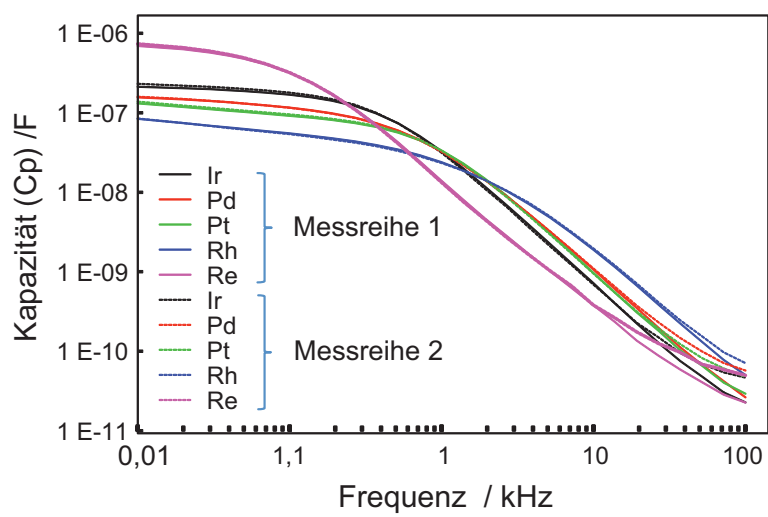

Abbildung 7:Kapazitiver Frequenzverlauf unterschiedlicher Elektrodenmaterialien, Elektrolyt Phosphatpuffer mit Zugabe von 0,5 g/L Propionsäure

Bei realen Messungen in Puffersystemen bzw. mit realen Biogasgüllen und einer großen Variation einzelner FOS (Tabelle 1) kann mit linearen Analysemethoden kein brauchbares Kalibrationsmodell zur Anwendung gebracht werden.

\section{Datenauswertung}

Die Datenerfassung mit 6 Elektroden liefert eine große Anzahl von Daten, welche weiter mit verschiedenen Methoden mathematisch analysiert werden. Ein Datensatz für eine Probe besteht je Elektrode aus minimal 36 Stützstellen je Spektrum (mit der Information: Zeit, Frequenz, Real- und Imaginärteil) mit 10 Wiederholungen.

Bei einer multivariaten Datenanalyse und Datenverarbeitung werden gegenüber einer univariaten Analyse mehrere Variablen gleichzeitig auf ihre Zusammenhänge und Abhängigkeiten untersucht und zur Generierung von Kalibrationsmodellen genutzt. Methoden der MSD kommen bevorzugt zum Einsatz, wo höherdimensionale Daten statistisch analysiert werden müssen.
In der Literatur zu ET $[3,4,5]$ wurden bereits eine ganze Reihe von Anwendungen der MSD beschrieben.

Besonders Regressionsanalysen (MLR: Multiple Linear Regression, PCR: Principal Component Regression, PLSR: Partial Least Squares Regression) sind gut geeignet, um Beziehungen zwischen einer abhängigen und einer oder mehreren unabhängigen Variablen quantitativ zu beschreiben. Die PLSR ist eine in der NahinfrarotSpektroskopie (NIRS) etablierte Methode.

Die Datenvorverarbeitung durch Bildung von Mittelwerten, Filterung und Anwendung von Methoden der MSD erfolgte mit MATLAB und diente u.a. zum Korrigieren der Basislinien, der Rauschunterdrückung, Erkennen von Ausreißern, Skalierung und Normalisierung.

Für die Erstellung eines Kalibrationsmodells erfolgte zunächst die Aufteilung in einen Trainings- und einen Testdatensatz. Im Rahmen dieser Arbeit wurde zur quantitativen Analyse eine PLSR durchgeführt, um ein modellbasiertes überwachtes Verfahren zur Anwendung zu bringen.

Tabelle 2: Mischungen von Essigsäure (ES) und Propionsäure (PS) in Phosphatpuffer

\begin{tabular}{|c|c|c|}
\hline Nummer & ES in $\mathbf{g} / \mathbf{I}$ & PS in $\mathbf{g} / \mathbf{I}$ \\
\hline 1 & 0.5 & 0.125 \\
\hline 2 & 0.5 & 0.25 \\
\hline 3 & 1 & 0.5 \\
\hline 4 & 1 & 1.5 \\
\hline 5 & 0.5 & 0.125 \\
\hline 6 & 0.5 & 0.25 \\
\hline 7 & 1 & 0.5 \\
\hline 8 & 1 & 1.5 \\
\hline 9 & 0.5 & 0.125 \\
\hline 10 & 0.5 & 0.25 \\
\hline 11 & 1 & 0.5 \\
\hline 12 & 1 & 1.5 \\
\hline
\end{tabular}

Abbildung 18 zeigt das Ergebnis einer Auswertung mit einem PLSR Modell. Dabei wurden vom Frequenzspektrum nur die Stützstellen bei $1 \mathrm{kHz}$ und $10 \mathrm{kHz}$ angewendet. In der Abbildung 8 sind die Messwerte zur Kalibration als offener größerer Kreis dargestellt. 


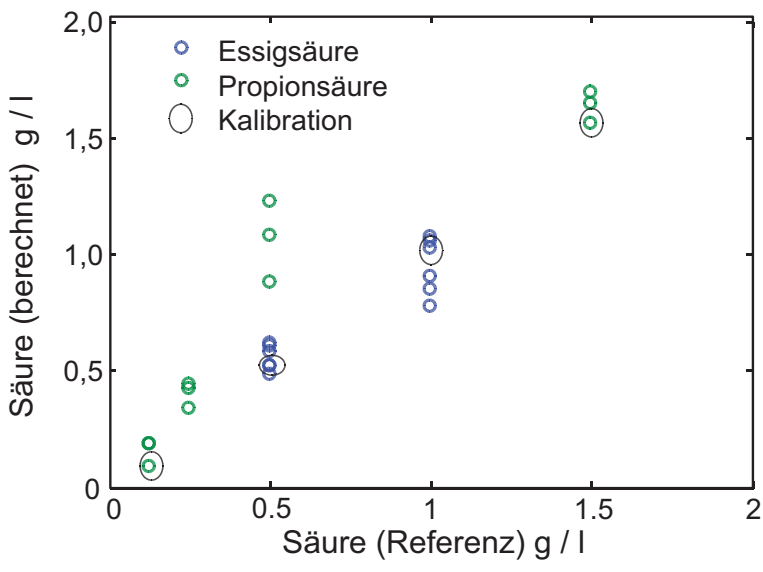

Abbildung 8: Ergebnis einer PLS

Die in der Abbildung 8 gezeigten Ergebnisse sind ein erstes Beispiel für die Anwendungen der impedanzspektroskopischen ET.

Die Ergebnisse mit den vorhergesagten Werten lassen sich durch Auswahl von Variablen aus dem gesamten Spektrum deutlich verbessern.

\section{Diskussion und Ausblick}

Es wurde gezeigt, dass durch die Anwendung einer ET mit impedanzspektroskopischer Auswertung der Anteil von VOC in Flüssigkeiten möglich ist. Die Konzentrationen der VOS entsprachen den in Biogasanlagen vorkommenden Konzentrationen (Tabelle 1).

Durch Anwendung von relevanten Ersatzschaltungen für die elektrochemischen Elektroden/ Elektrolytinteraktionen (Abbildung 4) zur frequenzabhängigen Merkmalsextraktion in Verbindung mit PLSR-Kalibrationsmodellen können zuverlässigere Vorhersagemodelle generiert werden.

Damit kann ein Nachteil der PLSR, welcher in der schlechten Interpretation des Modellansatzes besteht, überwunden werden. Eine Überführung des MATLAB Modells in eine funktionsfähige Runtime auf Basis einer einfachen Schaltung mit Mikrokontrollern wurde bereits im iba bei der Entwicklung einer online prozesstauglichen Mikrowellensonde zur Bestimmung des TS bzw. oTS an BGA realisiert [10].

Ein noch wesentlicher Schwachpunkt der bisherigen Messungen liegt in der Vermeidung von Verstopfungen in der Fluidstrecke. Feststoffe, welche zwangsläufig in realen Proben einer BGA vorliegen, müssen eliminiert werden.

Eine, für reale BGA nutzbare automatisierte Probennahme ist Gegenstand aktueller Entwicklungen. Damit wird eine wichtige Voraussetzung geschaffen, um ET auch an weiteren Anwendungen zur stofflichen und energetischen Nutzung von Biomasse anzuwenden.

\section{Literatur}

[1] J. Liebetrau, D. Pfeiffer, D. Thrän, Messmethodensammlung Biogas Methoden zur Bestimmung von analytischen und prozessbeschreibenden Parametern im Biogasbereich. Schriftenreihe des BMUFörderprogramms „Energetische Biomassenutzung (2015) Band 7: 213 Seiten

[2] B. Drosg, Process monitoring in biogas plants.
IEA
Bioenergy;
(2013)

ISBN 978-1-910154-03-8

[3] M. Peris, L. Escuder-Gilabert, On-line monitoring of food fermentation processes using electronic noses and electronic tongues: A review. Analytica Chimica Acta 804 (0) (2013) 29-36.

[4] A. Riul, C.A. R. Dantas, et al. Recent advances in electronic tongues. Analyst 135 (10) (2010) 2481-2495,

[5] F. P. A., Cabral, B. B. Bergamo, et al. Impedance e-tongue instrument for rapid liquid assessment. Review of Scientific Instruments 80(2) (2009) 26107-26107

[6] P. A Ulloa, et al. Determination of the botanical origin of honey by sensor fusion of impedance e-tongue and optical spectroscopy." Computers and Electronics in Agriculture 94(0) (2013)1-11

[7] H. Hamann, W. Vielstich, Elektrochemie IIElektrodenprozesse, angewandte Elektrochemie, Verlag Chemie, Weinheim, (1981).

[8] E. Barsoukov, J.R. Macdonald, Impedance Spectroscopy: Theory, Experiment, and Applications. Wiley-Interscience. (2005) ISBN: 0-471-64749-7

[9] J.-P. Diard, C. M. B. Le Gorrec, FARADAIC IMPEDANCE LIBRARY. Handbook of Electrochemical Impedance Spectroscopy. (2009)

[10] T. Nacke, A. Göller, Verbundvorhaben: Entwicklung einer prozesstauglichen OnlineMessanordnung zur Bestimmung der organischen Trockensubstanz in Biogasanlagen. Abschlussbericht, FNR Förderkennzeichen FKZ 22017608, (2007)

Danksagung: Die Arbeiten werden im Rahmen eines Verbundprojektes durch das BMBF, Projektträger Jülich, gefördert (Förderkennzeichen 305 ZBG). Der Titel lautet: „Online-Prozessanalytik qualitätsbeeinflussender Parameter bei der Biogasproduktion durch intelligente Sensorik". 